\title{
Price-Reward for Data Relaying and Handover Management in Wireless Networks
}

\author{
Muhammad Shoaib Saleem \\ Institut Télécom - Télécom SudParis \\ 9, Rue Charles Fourier \\ Évry, France \\ shoaib.saleem@it-sudparis.eu
}

\author{
Éric Renault \\ Institut Télécom - Télécom SudParis \\ 9, Rue Charles Fourier \\ Évry, France \\ eric.renault@it-sudparis.eu
}

\begin{abstract}
We propose Price-Reward for Data Relaying and Handover in Wireless Networks, a handover and data relay management algorithm for wireless networks. The algorithm works in dynamic environment in which mobile nodes move randomly. The mathematical model presented explains how cooperative diversity helps to augment network coverage plus revenue and ensures connection reliability during mobility and handover situations.
\end{abstract}

\section{Categories and Subject Descriptors}

C.2.1 [Network Architecture and Design]: Wireless communication

\section{General Terms}

Algorithms

\section{Keywords}

Wireless Networks, Stackelberg Model, Price-Reward

\section{INTRODUCTION}

The use of the Internet in wireless networks always demand for better QoS during mobility. A wide range of proposal suggest either to adopt high performance protocols or replace the contemporary Internet architecture with a new one that incorporates all the desired features required today. Seamless mobility in wireless networks is an issue addressed widely. We, in [3], proposed an mobile node architecture for the Future Internet called Network of Information Mobile Node (NetInf Mobile Node). It possesses features like seamless handover and data relaying during mobility to sustain the QoS during an ongoing session.

However, in order to analyze its performance, the two possible methods are the analytical and the simulation approaches. In an analytical approach, a mathematical model is developed with the help of some available tools. For example, in this paper we are using Game Theory. In an environment, where nodes do not have complete information about their network and are moving randomly, the learning schemes in [4] can aide mobile nodes to update their strategies and utility functions. The cooperative diversity

Copyright is held by the author/owner(s).

MobiHoc'12, June 11-14, 2012, Hilton Head, South Carolina, USA.

ACM 978-1-4503-1281-3/12/06. of the network is ensured by using Stackelberg model approach where an Access Point $(A P)$ introduces the price $(\lambda)$-reward $(\mu)$ parameter. The prime objective of the game presented in this work is that all the players maximize their utilities or payoff. The algorithm which is developed using these techniques is named Price-Reward for Data Relaying and Handover in Wireless Networks.

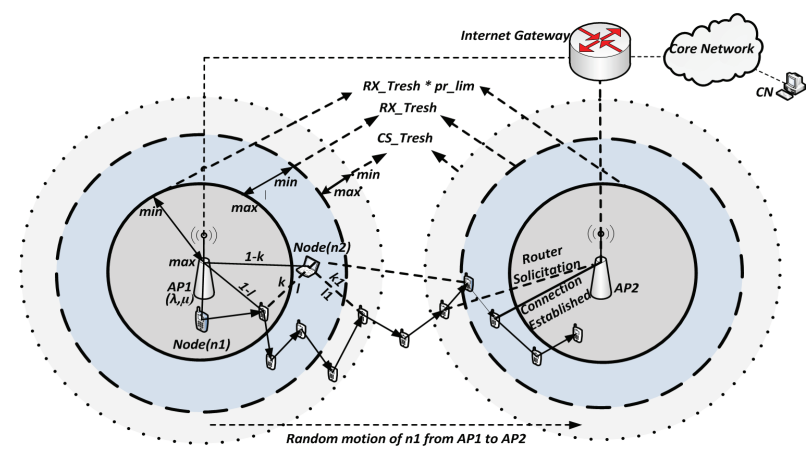

Figure 1: Data Relay and Handover

\section{THE ALGORITHM}

The working principle of our algorithm is explained with the help of a scenario illustrated in the Figure 1. The system has two wireless Access Points $A P 1$ and $A P \mathscr{2}$, two mobile nodes, $n 1$ and $n 2$ and a Corresponding Node $C N$. We assume that $n 1$ is in session with $C N$. The area covered by an $A P$ is divided into three levels as defined in [1]. The three levels are (RX_Tresh * pr_lim), (RX_Tresh) and (CS_Tresh) with minimum and maximum threshold values as shown.

The algorithm performs its functions in three phases after being initialized by the network when the $(\lambda, \mu)$ values are advertised. The first phase involves the ( $R X_{-}$Tresh) level where after some time $t, n 1$ starts moving away from $A P 1$. As a result the $R S S$ (Received Signal Strength) value decreases in $\left(R X \_\right.$Tresh ${ }^{*}$ pr_lim) level where $\left(0 \leq p r \_l i m \leq 1\right)$ and defines the minimum and maximum $R S S$ values. This triggers the data relaying process. Node $n 1$ allocates $l$ bits of its data stream to be relayed by a neighboring node. It broadcasts packets carrying data forwarding request. Mobile Node $n 2$, which has already updated itself with $(\lambda, \mu)$, acknowledges $n 1$ request by proposing $k$ fraction of its channel to relay $n 1$ 's $l$ bits of data stream. $n 1$ upon receiving the reply, informs $A P 1$ and directs $l$ bits of data stream towards n2. The direct link between $n 1$ and $A P 1$ carries (1-l) bits of 
data and $n 2$ uses (1-k) fraction of its channel to stream its own data.

The second phase starts when the $R S S$ becomes minimum in ( $R X_{-}$Tresh) level and the probability of packet loss between $n 1$ and $A P 1$ link increases. In such circumstances, $n 1$ finds it hard to traffic its data via direct link with $A P 1$. At this moment, the network announces new $(\lambda, \mu)$ values with increased incentive $(\mu)$ for relaying nodes ( $n 2$ in our case). Following that, $n 1$ diverts most of its data stream, say $l_{1}$ $\mid l_{1}>l$ to the link between $n 1$ and n2. n2 updates $(\lambda, \mu)$ information and allocates fraction of its channel, $k_{1} \mid k_{1}>k$, for $n 1$ 's $l_{1}$ data stream. Once $n 1$ enters (CS_Tresh) level, $n 2$ starts relaying most of the $n 1$ data stream.

The final phase starts when the $R S S$ value in (CS_Tresh) level gets equal to zero. The link between $n 1$ and $A P 1$ break-offs. This critical phase lasts when eventually $N 1$ detects (CS_Tresh) level of AP2. In (RX_Tresh) ${ }_{\text {min }}$ of AP2, n1 sends a router solicitation message to AP2. AP2 checks the $n 1$ authenticity with the help of Internet gateway and sends back router advertisement. While accepting the request of stronger connection, $n 1$ requests $A P 1$ via $n 2$ to inform Internet gateway to detour $C N$ traffic towards $A P$ 2. Once the connection between $n 1$ and $A P 2$ establishes, $n 1$ terminates its link with n2. The acknowledgement of route diversion is received via $A P 2$.

\section{THE SYSTEM MODEL}

Let $\mathcal{N}$ be the set of mobile nodes in a wireless network. From [4] we adopt a Combined fully Distributed Payoff and Strategy Reinforcement Learning (CODIPAS-RL) scheme for mobile nodes to define a strategy that can help to maximize their utility functions. Each mobile node has a set of finite action. For example, we consider a scenario of two mobile nodes $(n 1, n 2)$ with $\mathcal{A}_{1}$ and $\mathcal{A}_{2}$ be the sets of finite number of actions in each respectively. Based on each action, a mobile node updates its strategy and payoff functions. Let $a_{n_{i}}$ be an action chosen by any node $n_{i} \mid i \in \mathcal{N}$. The learning rates $r_{n_{i}, t}$ and $q_{n_{i}, t}$ are assigned for the strategy update $\left(x_{n_{i}, t+1}\right)$ and estimated payoff $\left(\hat{u}_{n_{i}, t+1}\left(a_{n_{i}}\right)\right)$ functions respectively as shown in eq.(1). $\mathbb{I}_{a_{n_{i}, t}=a_{n_{i}}}$ is an indicator function for the chosen action $a_{n_{i}}$ at time $t$ and $U_{n_{i}, t}$ is the observed utility function for any node $n_{i}$.

$$
\begin{aligned}
& x_{n_{i}, t+1}=x_{n_{i}, t}\left(a_{n_{i}}\right)+r_{n_{i}, t} U_{n_{i}, t}\left(\mathbb{I}_{a_{n_{i}, t}=a_{n_{i}}}-x_{n_{i}, t}\left(a_{n_{i}}\right)\right) \\
& \hat{u}_{n_{i}, t+1}\left(a_{n_{i}}\right)=\hat{u}_{n_{i}, t}\left(a_{n_{i}}\right)+q_{n_{i}, t} \mathbb{I}_{a_{n_{i}, t}=a_{n_{i}}}\left(U_{n_{i}, t}-\hat{u_{n_{i}, t}}\right)
\end{aligned}
$$

We consider a network with finite number of states for a node $n_{i}$ represented by the set $\mathcal{S}_{i}$ in which $s_{n_{i}, j}$ is a state for any node $n_{i}$ where $j=(1,2, \ldots . M)$. Using $U_{n_{i}, t}$, the strategy selected at time $t$ with $a_{n_{i}}$ as the chosen action, is $x_{n_{i}, t}\left(a_{n_{i}}\right)$ and the strategy function $x_{n_{i}, t+1}$ is updated using eq.(1). The estimated utility $\hat{u}_{n_{i}, t+1}\left(a_{n_{i}}\right)$ function is also updated through the same procedure.

Eq.( 2) \& eq.( 3) represents $n 1 \& n 2$ utility functions where $P_{n 1} \& P_{n 2}$ are their powers respectively. $f($.$) is the efficiency$ function from [2] and $\gamma$ is the SNR. For three different paths we have $\gamma_{n 1 n 2}, \gamma_{n 1 a 1}$ and $\gamma_{n 2 a 1} . n 1$ and n2 optimize their utilities using eq.(2) \& eq.(3).

$$
\begin{gathered}
\text { Node } 1: \max U_{n 1}\left(P_{n 1}, l\right) \\
\equiv \max \left(\frac{1}{P_{n 1}}-\lambda\right)\left[(1-l) f\left(\gamma_{n 1 a 1}\left(P_{n 1}\right)\right)+l f\left(\gamma_{n 1 n 2}\left(P_{n 1}\right)\right)\right. \\
\text { s.t. }\left\{\begin{array}{l}
0 \leq P_{n 1} \leq P^{\max }, 0 \leq l \leq 1, \\
l f\left(\gamma_{n 1 n 2}\left(P_{n 1}\right)\right) \leq k f\left(\gamma_{n 2 a 1}\left(P_{n 2}\right)\right) .
\end{array}\right. \\
\text { Node } 2: \max U_{n 2}\left(P_{n 2}, k\right) \\
\text { इmax }\left[(1-k)\left(\frac{1}{P_{n 1}}-\lambda\right)+k(\mu-\lambda)\right] f\left(\gamma_{n 2 a 1}\left(P_{n 2}\right)\right) \\
\text { s.t. }\left\{\begin{array}{l}
0 \leq P_{n 2} \leq P^{\max }, 0 \leq k \leq 1, \\
l f\left(\gamma_{n 1 n 2}\left(P_{n 1}\right)\right) \geq k f\left(\gamma_{n 2 a 1}\left(P_{n 2}\right)\right) .
\end{array}\right.
\end{gathered}
$$

For every $(\lambda, \mu)$ pair the two nodes compete to maximize their $U_{n_{i}, t}$ using eq.(2) \& eq.(3) for different strategies in eq.(1). Once the point of equilibrium is achieved, the network utilizes the equilibrium values $\left(P_{n 1}^{*}, l^{*}\right)$ and $\left(P_{n 2}^{*}, k^{*}\right)$ of $n 1, n 2$ and calculates its net-revenue $R_{\text {net }}$ using eq.(4) for different values of $(\lambda, \mu)$.

$$
R_{n e t}\left[\left(P_{n 1}^{*}, l^{*}\right),\left(P_{n 2}^{*}, k^{*}\right)\right]=\max _{(\lambda \geq=0, \mu \geq=0)} R
$$

where $R$ is,

$$
R=\sum \lambda\left(\text { Throughput }_{\text {allnodes }}\right)-\sum \mu\left(\text { Throughput }_{\text {relayingnodes }}\right)
$$

\section{CONCLUSION AND FUTURE WORK}

We proposed a data relaying and handover management algorithm for wireless networks. The mathematical model discussed explains the strategy selection and utility maximization methods. We are currently using NS-2 patch developed by NIST [1] (for heterogeneous wireless networks) for implementation and performance evaluation for our algorithm. The robustness of the algorithm will be proved by showing how quickly it can converge to an equilibrium state when a mobility or a handover scenario is taken into account.

\section{REFERENCES}

[1] "The Network Simulator NS-2 - NIST add-on - Mac 802.11", National Institute of Standards and Technology (NIST), January 2007.

[2] D. Goodman and N. Mandayam. Power control for wireless data. IEEE International Workshop on Mobile Multimedia Communications, (MoMuC '99), 1999.

[3] M. Saleem, E. Renault, and D. Zeghlache. Netlnf Mobile Node Architecture and Mobility Management Based on Lisp Mobile Node. IEEE Consumer Communications and Networking Conference (CCNC), 2011.

[4] Q. Zhu, H. Tembine, and T. Basar. Distributed Strategic Learning with Application to Network Security. American Control Conference (ACC), 2011. 